\title{
Uranium speciation models in contaminated soils
}

\author{
ANNA KROT ${ }^{1}$, IRINA E. VLASOVA ${ }^{2}$ AND ALEXANDER \\ TRIGUB $^{3}$ \\ ${ }^{1}$ Lomonosow Moscow State University \\ ${ }^{2}$ Lomonosov Moscow State University \\ ${ }^{3}$ NRC Kurchatov Institute \\ Presenting Author: Anna.d.krot@gmail.com
}

In common environmental conditions, i.e. in presence of oxygen and $\mathrm{CO} 2$, uranium is known to form high-mobile $\mathrm{UO} 22+$ soluble species. Immobilization includes transformation of these complex species into insoluble forms in precipitation/coprecipitation, sorption and reduction processes. Therefore, it is of high importance to understand the speciation of Uranium in various geochemical conditions that occurred in contaminated sites. One of the most useful non-destructive method to obtain information on local surrounding is U LIII-edge X-Ray adsorption spectroscopy. Based on the accumulated spectral data, one can design an appropriate $U$ speciation model in contaminated soils, which is crucial for developing remediation strategies.

In this work, we focused on the determining of uranium speciation in the most contaminated fractions of the soil of the Angarsk Electrochemical Combine (AEC) by using complex of methods: XRD-SR, XAFS, SEM, XRF, $\mu$-XRF. When interpreting the EXAFS spectra, we relied on the results for labprepared reference samples: synthesized compounds of U6+ and model sorption species. According to XANES data, all studied soil samples contained uranium in the form of uranyl UO22+. Based on the obtained data on the nature, interatomic distance, and coordination numbers of the atoms of $U$ nearest surrounding, speciation models of U(VI) in studied objects were proposed. It was found that speciation of uranium, even within the same soil, is strongly depend on the nature of each individual component of the soil. A common feature of local surrounding of most objects is the presence of $\mathrm{C}$ atoms at $\mathrm{R}$ - distance of $\sim 2.9 \AA$, reflecting carbonates or NOM-molecules coordinated to U(VI). For coal and plant residues, neighboring $U$ atoms were found at a distance of $\sim 3.7 \AA$, which is typical for schoepite-like structures. It was observed that in Fe-slag and wood residues samples sorption processes predominates over precipitation of U-bearing phases. In clay fraction, the formation of schoepite-like species and complexes with carbon in the nearest coordination sphere carbonates or NOM-molecules - was revealed. Obtained EXAFS data and proposed speciation models will help to carry out procedures for rehabilitation of U-contaminated soils.

This work was financially supported by the Russian Science Foundation (grant No19-73- 20051). 